\title{
Central blood pressure in adults screened for a genetic polymorphism in folate metabolism
}

\section{Abstract}

As the primary risk factor for cardiovascular disease (CVD), hypertension is the leading cause of preventable, premature mortality globally. Hypertension, or elevated blood pressure (BP), has a number of well-established risk factors, including genetics. A common C677T polymorphism in the gene encoding the folate metabolising enzyme methylenetetrahydrofolate reductase (MTHFR) affects $10-12 \%$ of UK and Irish populations and has been linked with $24-87 \%$ increased risk of hypertension globally. Evidence from randomised controlled trials (RCTs) conducted at this Centre has shown BP to be highly responsive (by 5-13 $\mathrm{mmHg}$ ) to supplementation with riboflavin (MTHFR co-factor), an effect confined to homozygous individuals (TT genotype). To date, our trials have focused on peripheral BP; however, additional measures of vascular health such as central pressure are reported to be more closely correlated with CVD risk. Investigation of central BP, augmentation index (AIx) and pulse pressure amplification (PPA) may thus offer further insight into the role of this gene-nutrient interaction in blood pressure. The present study aims to investigate BP, and measures of vascular health in healthy adults stratified by MTHFR 677 genotype. Apparently healthy adults aged 18-60 years were recruited from workplaces across Northern Ireland and screened for MTHFR genotype via buccal swab. Clinic BP, anthropometry and blood sample were measured in TT individuals $(n$ 209) and age and sex-matched CC $(n$ 98) and CT $(n$ 102) controls. AIx and central BP were assessed using SphygmoCor ${ }^{\circledR}$ (AtCor Medical, Australia). Preliminary results demonstrate higher BP in individuals with the MTHFR 677TT genotype compared to non-TT controls (systolic BP $134.7 \pm 13.8 \mathrm{mmHg}$ vs $129.7 \pm 12.4 \mathrm{mmHg}, \mathrm{P}<0.001 ;$ diastolic BP $81.6 \pm 9.5 \mathrm{mmHg}$ vs $79.7 \mathrm{mmHg} \pm 8.9 \mathrm{mmHg}, \mathrm{P}=0.023$, respectively). The MTHFR $677 \mathrm{TT}$ genotype group had significantly higher central systolic $\mathrm{BP}(119.4 \pm 11.8$ vs $116.7 \pm 10.9 \mathrm{mmHg}, \mathrm{P}=0.018)$, central pulse pressure $(\mathrm{P}=0.006)$ and central mean pressure $(\mathrm{P}=0.011)$ compared to the non-TT group. No significant differences for central diastolic BP, pulse pressure amplification, pulse pressure ratio and augmentation index were observed. This study confirms the phenotype of elevated BP in individuals with the C677T polymorphism in the gene encoding MTHFR. For the first time, this study reports that individuals with the MTHFR 677TT genotype have higher central systolic BP, central mean pressure and pulse pressure. Further investigations through RCTs investigating the effect of the MTHFR cofactor, riboflavin, on central blood pressure in these genetically at-risk adults are warranted.

\section{Conflict of Interest}

There is no conflict of interest 